\title{
Anesthetics and cerebral ischemia - should we continue to dream the impossible dream?
}

\author{
Adrian W. Gelb MD, ${ }^{*}$ John X. Wilson PhD, $†$ David F. Cechetto PhD $\ddagger$
}

A

therapeutic role in cerebral ischemia for anesthetics has been the subject of fluctuating interest for the past $40 \mathrm{yr}$. This interest seemed to be driven by the beguilingly simple idea that anesthetics reduce metabolic requirements and therefore should prolong the duration of time that the brain can tolerate a reduction in oxygen delivery. At a subliminal level, there may also have been an interest by anesthesiologists, whose practice is one of "service", to find a therapeutic role for themselves. An influential editorial by Todd and Warner in 1992 made the cogent argument that metabolic suppression alone did not adequately explain an anesthetic protective effect, if one existed at all. ${ }^{1}$ This seemed to be followed by a substantial dissipation of general interest in the subject except by stalwart investigators.

The effects of anesthetics on the ischemic brain however are not just a question of interest or importance for the researcher. Whenever we administer anesthesia to a patient in whom brain ischemia may occur - neurosurgery, cardiac surgery, surgery of or around the carotid and vertebral arteries, use of induced hypotension - we have an obligation to know that the choice of drugs will not worsen ischemia should it occur. A finding that an anesthetic or drug regimen does not worsen outcome should therefore be seen as an important and desirable finding.

A related question, but not pertinent to all circumstances, is whether the anesthetized state is better than being awake at the time of ischemic risk. Early studies, using crude outcomes such as survival, indicated that any available anesthetic except nitrous oxide was better than no anesthetic. ${ }^{2}$ Ethical and methodological issues prevented this issue being addressed with focal ischemia, which is more clinically relevant, until the recent development of techniques to produce focal ischemia in the awake animal. Using a variety of methods, halothane, isoflurane, sevoflurane and propofol have all been shown to reduce infarct size relative to the awake state. ${ }^{3-6}$ An ongoing randomized trial of general $v s$ regional anesthesia for carotid endarterectomy, the GALA trial, may help determine the clinical relevance of these findings.

Having determined that anesthetics do not worsen outcome, is there is any reason to study them further? Unequivocally yes, as many questions remain unanswered. There is a need to determine if there is a preferred choice for the patient at risk of intraoperative cerebral ischemia. Interpretation of the laboratory literature is made difficult by the extensive use of surrogate endpoints such as biochemistry or electrophysiology rather than infarct size or neurological outcome. A cautious interpretation of the experimental literature indicates that neither nitrous oxide nor a narcotic anesthetic offers measurable protection. ${ }^{5}$ The currently used inhalation agents appear similar to each other and propofol is similar or possibly better. Although negative studies exist, the weight of the literature on thiopentone suggests that it is better than the inhalational agents. ${ }^{7}$ Unfortunately, there are very few human prospective randomized trials and most of those are in the context of various cardiac surgery protocols that offer a high incidence of measurable neuropsychological outcomes but are not necessarily appropriate to extrapolate to other clinical circumstances. ${ }^{8,9}$

Other important issues are timing of drug administration, dose, durability of effect, and mechanism of action. The experimental literature is consistent in finding that anesthetics work best if given early in the ischemic period or, preferably, prophylactically. Just how far in advance is a new and exciting question as a recent report indicates that isoflurane given for an hour a day substantially reduces the size of a subsequent infarct not induced under anesthesia; this is an example of anesthetic-induced preconditioning. ${ }^{10}$ The appropriate dose or anesthetic depth has also been debated over the decades. The belief was that a dose that achieved EEG burst suppression (i.e., maximal 
metabolic suppression), would be best but the realization that anesthetic protection is more than metabolic suppression has challenged this notion. ${ }^{11}$ Recent developments of animal models that allow prolonged follow-up have demonstrated that many "protective agents", including isoflurane, may only produce an early benefit that dissipates over a period of weeks. ${ }^{12}$

There is also a need to better understand the mechanisms by which our drugs improve or worsen ischemia. Not only may this provide some insights into the mechanisms of anesthesia but it may also lead to the development of better anti-ischemic drugs, which would have similar mechanisms of action but not induce unconsciousness. Although metabolic suppression of the brain by any means may induce some tolerance of ischemia, such a notion does not advance our understanding or explain the range of effects of different anesthetics. Anesthetic agents are a diverse group of compounds with the capability of producing many simultaneous extracellular and intracellular effects. It would be much more useful to think of each agent as producing a set of unique effects, although there will likely be some overlap between agents. As potentiation of the inhibitory transmitter GABA is an effect common to many anesthetics and GABA has been shown to reduce infarct size, this could explain an effect common to most inhaled and iv anesthetic agents and also the reduction in metabolism. ${ }^{13}$ In addition, agents could have multiple other effects including antioxidant defense, alteration of calcium and sodium fluxes, inhibition of excitatory neurotransmission, etc.

The complexity that may be associated with each mechanism may be further illustrated by examining the multifactorial ways by which anesthetics could modulate oxidative stress (i.e., an excessive rate of free radical production relative to elimination) and prevent the irreversible injury caused by reactive oxygen and nitrogen species. ${ }^{14}$ Oxidative stress occurs in the brain during ischemia-reperfusion. By slowing the cerebral utilization of oxygen in brain parenchymal cells, anesthetics may attenuate the intracellular generation of free oxygen radicals. Anesthetic modulation of glutamate release, reuptake and receptor activity may also retard the calcium-mediated production of oxidizing radicals within neurons. Furthermore, anesthetics diminish free radical production by activated neutrophils, which contribute to brain oxidative stress during ischemia and reperfusion. Finally, anesthetics such as thiopentone and propofol are reductants that potently scavenge reactive oxygen and nitrogen species; in other words, they react preferentially with oxidizing radicals to yield relatively stable products. By donating electrons to free radicals, these antioxi- dant anesthetics spare other molecules from oxidation; for example, they prevent lipid peroxidation in cell membranes and lipoproteins.

In this issue of the Journal, Cole et al. ${ }^{15}$ advance our understanding by addressing some of the issues alluded to above. By comparing three barbiturates at two different doses each, a burst suppression dose and $40 \%$ thereof, they found that thiopentone at burst suppression and methohexital at $40 \%$ of a burst suppression dose significantly reduced infarct volume compared to the other doses, pentobarbitone at both doses and halothane. They have nicely confirmed: i) that more than simple metabolic suppression is involved, ii) the importance of drug choice and dose, and iii) that one cannot simply extrapolate from one drug to another.

Much has been learned about cerebral ischemia and the effects of anesthetics. However, if, when, how much and how long to use them remains poorly defined. A therapeutic use for anesthetics in cerebral ischemia remains a dream, but a dream with an increasingly rational basis and therefore an impossible dream worthy of ongoing pursuit.

\section{Les anesthésiques et l'ischémie cérébrale - poursuivre l'impossible rêve?}

Le rôle thérapeutique des anesthésiques dans l'ischémie cérébrale n'a pas toujours suscité le même intérêt au cours des 40 dernières années. Cet intérêt a semblé stimulé par l'idée simple et attirante que les anesthésiques pouvaient réduire les besoins métaboliques et, donc, prolonger la durée pendant laquelle le cerveau pouvait tolérer une réduction de l'apport en oxygène. Peut-être y at-il eu aussi, inconsciemment, de la part des anesthésiologistes dont la pratique est un travail de "service", le désir de se découvrir un rôle thérapeutique. Un éditorial influent de Todd et Warner, publié en 1992, a présenté l'argument convaincant voulant que la suppression métabolique seule ne puisse expliquer entièrement l'effet protecteur d'un anesthésique, si jamais il en existait un. ${ }^{1}$ Cette assertion a été suivie, semble-t-il, par une dissipation importante de l'intérêt général pour le sujet, sauf chez les chercheurs résolus. 
Les effets des anesthésiques sur le cerveau ischémique ne sont cependant pas une question d'intérêt ou d'importance pour le chercheur seulement. Chaque fois que nous administrons une anesthésie à un patient chez qui l'ischémie cérébrale pourrait survenir - que ce soit en neurochirurgie, cardiochirurgie, chirurgie de la carotide et des artères vertébrales ou autour de ces artères, utilisation d'hypotension induite - nous devons savoir si les médicaments choisis aggraveront l'ischémie potentielle. La découverte d'un anesthésique ou d'un régime posologique qui ne compliquerait pas l'évolution serait donc une découverte importante et souhaitable.

La question reliée, mais non pertinente en toutes circonstances, est de savoir si c'est préférable d'être anesthésié ou éveillé au moment où il y a risque d'ischémie. Des études antérieures, fondées sur des issues imprécises comme la survie, ont indiqué que tout anesthésique disponible, sauf le protoxyde d'azote, était meilleur que l'absence d'anesthésique. ${ }^{2}$ Des questions d'éthique et de méthodologie ont empêché que ce problème soit abordé dans le cas de l'ischémie focale, qui concerne davantage la pratique clinique, jusqu'à la récente mise au point de techniques permettant de produire l'ischémie focale chez un animal éveillé. Utilisés selon diverses méthodes, l'halothane, l'isoflurane, le sévoflurane et le propofol ont réduit l'importance de l'infarctus à l'état d'éveil. ${ }^{3-6}$ Un essai randomisé en cours sur l'anesthésie générale vs l'anesthésie régionale pour l'endartériectomie de la carotide, l'essai GALA, pourrait contribuer à déterminer la pertinence clinique de ces constatations.

Ayant montré que des anesthésiques ne compliquent pas l'évolution, existe-t-il une raison d'en faire une étude plus poussée? A l'évidence, oui, puisque de nombreuses questions demeurent sans réponse. Ainsi, il faut déterminer s'il y a une méthode préférable pour le patient à risque d'ischémie cérébrale peropératoire. L'interprétation des études de laboratoire est rendue difficile par l'usage étendu de paramètres substituts comme ceux qui relèvent de la biochimie ou de l'électrophysiologie plutôt que du volume de l'infarctus ou de l'évolution neurologique. Une interprétation prudente des données expérimentales indique que ni le protoxyde d'azote, ni un anesthésique narcotique n'offrent de protection mesurable. ${ }^{5}$ Les agents d'inhalation les plus courants se ressemblent, le propofol est semblable ou peut-être meilleur en terme de protection. Bien que des études négatives existent, l'abondance de données sur le thiopental laisse croire qu'il est meilleur que les agents d'inhalation. ${ }^{7}$ Malheureusement, il y a peu d'essais prospectifs randomisés chez l'humain et la majorité a été réalisée dans le contexte de divers protocoles de cardiochirurgie à forte incidence d'effets neuropsychologiques mesurables mais qui ne se prêtent pas nécessairement à une extrapolation à d'autres situations cliniques. ${ }^{8,9}$

D'autres aspects importants concernent le moment choisi pour administrer le médicament, la dose, la durabilité de l'effet et le mécanisme d'action. Les données expérimentales montrent généralement que les anesthésiques agissent mieux s'ils sont administrés au début de l'ischémie ou, de préférence, de façon prophylactique. Mais combien de temps d'avance, c'est une question nouvelle et enthousiasmante alors qu'un récent article indique que l'isoflurane donné une heure par jour réduit considérablement l'importance d'un infarctus ultérieur non induit sous anesthésie; c'est un exemple de préconditionnement induit par un anesthésique. ${ }^{10} \mathrm{La}$ dose requise ou la profondeur de l'anesthésie ont aussi fait l'objet de débats pendant des décennies. On croyait qu'une dose qui supprimerait les bouffées du tracé EEG (c.-à-d., une suppression métabolique maximale) serait la meilleure, mais on s'est rendu compte que la protection anesthésique c'est plus que la suppression métabolique, ce qui a remis cette notion en cause. ${ }^{11}$ Les progrès récents des modèles animaux qui ont permis de prolonger l'observation ont démontré que de nombreux "agents protecteurs", y compris l'isoflurane, ne peuvent produire qu'un bénéfice précoce qui disparaît après quelques semaines. ${ }^{12}$

Il faut aussi comprendre mieux les mécanismes par lesquels nos médicaments améliorent ou aggravent l'ischémie. Ces connaissances pourraient fournir non seulement un aperçu des mécanismes de l'anesthésie, mais pourraient conduire aussi à fabriquer de meilleurs médicaments anti-ischémie dont les mécanismes d'action pourraient être similaires sans provoquer l'inconscience. La suppression métabolique du cerveau par quelque moyen que ce soit induit une certaine tolérance à l'ischémie, mais cette idée n'apporte aucune connaissance nouvelle et ne définit pas l'étendue des effets de différents anesthésiques. Les anesthésiques sont un groupe de composés divers capables de produire de nombreux effets extracellulaires et intracellulaires simultanés. Il serait beaucoup plus valable de considérer chaque médicament comme produisant un ensemble d'effets uniques, malgré la probabilité d'un certain chevauchement entre les médicaments. Comme la potentialisation du neurotransmetteur inhibiteur GABA est un effet commun de nombreux anesthésiques et que GABA peut réduire l'infarctus, cela pourrait expliquer un effet commun de la plupart des anesthésiques d'inhalation et $i v$ et aussi 
la réduction du métabolisme. ${ }^{13}$ En outre, les anesthésiques peuvent présenter de nombreux autres effets, y compris la défense antioxydante, la modification des flux de calcium et de sodium, l'inhibition de la neurotransmission excitatrice, etc.

La complexité qui peut être associée à chacun des mécanismes sera mieux illustrée en vérifiant les moyens multifactoriels qu'empruntent les anesthésiques pour moduler le stress oxydatif (c.-à-d., un taux excessif de production de radicaux libres en regard de l'élimination) et prévenir une lésion irréversible causée par les composés oxygénés et azotés réactifs. ${ }^{14}$ Le stress oxydatif survient dans le cerveau pendant l'ischémie-reperfusion. En diminuant l'utilisation cérébrale d'oxygène dans les cellules parenchymateuses cérébrales, les anesthésiques peuvent réduire la génération intracellulaire de radicaux libres d'oxygène. La modulation anesthésique de la libération, du recaptage et de la réception du glutamate peut aussi retarder la production de radicaux oxydants d'origine calcique à l'intérieur des neurones. De plus, les anesthésiques diminuent la production de radicaux libres par les neutrophiles activés, lesquels contribuent au stress oxydatif cérébral pendant l'ischémie et la reperfusion. Finalement, les anesthésiques comme le thiopental et le propofol sont des substances réductrices qui épurent efficacement les composés d'oxygène et d'azote; autrement dit, ils réagissent de préférence avec les radicaux oxydants pour donner des produits relativement stables. En abandonnant des électrons aux radicaux libres, ces anesthésiques antioxydants préservent d'autres molécules de l'oxydation; ainsi, ils empêchent la peroxydation lipidique dans les membranes cellulaires et les lipoprotéines.

Dans le présent numéro du Journal, Cole et coll. ${ }^{15}$ font avancer nos connaissances en abordant certains des sujets mentionnés plus haut. En comparant trois barbituriques selon deux doses différentes, l'une qui cause la suppression des bouffées du tracé EEG et l'autre qui équivaut à $40 \%$ de la suppression, ils ont trouvé que le thiopental à une dose de suppression et le méthohexital à une dose de $40 \%$ de suppression ont significativement réduit le volume de l'infarctus comparativement à d'autres doses, aux deux doses de pentobarbital et à l'halothane. Ils ont minutieusement confirmé : i) qu'il y a plus que la simple suppression métabolique d'impliquée, ii) que le choix du médicament et de la dose est important et iii) qu'on ne peut tout simplement extrapoler les données d'un médicament à l'autre.

On connaît beaucoup de choses sur l'ischémie cérébrale et sur les effets des anesthésiques. Mais on n'en connaît pas encore très bien tous les si, quand, combien et comment de l'utilisation. Un usage thérapeutique des anesthésiques pour l'ischémie cérébrale demeure un rêve, mais un rêve qui a un fondement de plus en plus rationnel et, par conséquent, un impossible rêve digne d'une autre quête.

\section{References}

1 Todd MM, Warner DS. A comfortable hypothesis reevaluated. Cerebral metabolic depression and brain protection during ischemia (Editorial). Anesthesiology 1992; 76: 161-4.

2 Bain JA, Catton DV, Cox JMR, Spoerel WE. The effect of general anaesthesia on the tolerance of cerebral ischaemia in rabbits. Can Anaes Soc J 1967; 14: 69-78

3 Warner DS, McFarlane C, Todd MM, Ludwig P, McAllister AM. Sevoflurane and halothane reduce focal ischemic brain damage in the rat. Possible influence on thermoregulation. Anesthesiology 1993; 79: 985-92.

4 Warner DS, Ludwig PS, Pearlstein R, Brinkbous AD. Halothane reduces focal ischemic injury in the rat when brain temperature is controlled. Anesthesiology 1995; 82: 1237-45.

5 Soonthon-Brant V, Patel PM, Drummond JC, Cole DJ, Kelly PJ, Watson M. Fentanyl does not increase brain injury after focal cerebral ischemia in rats. Anesth Analg 1999; 88: 49-55.

6 Cechetto DF, Gelb AW. Propofol sedation compared to awake reduces cerebral infarct size in rats. Anesth Analg 1999; 88: S1-424 (abstract).

7 Gelb AW, Floyd P, Lok P, Peerless SJ, Farrell M. A prophylactic bolus of thiopentone does not protect against prolonged focal cerebral ischaemia. Can Anaesth Soc J 1986; 33: 173-7.

8 Roach GW, Newman MF, Murkin JM, et al. Ineffectiveness of burst suppression therapy in mitigating perioperative cerebrovascular dysfunction. Anesthesiology 1999; 90: 1255-64.

9 Nussmeier NA, Arlund C, Slogoff S. Neuropsychiatric complications after cardiopulmonary bypass: cerebral protection by a barbiturate. Anesthesiology 1986; 64: 165-70.

10 Xiong L, Zhu Z, Dong H, et al. Isoflurane preconditioning induces neuroprotection against middle cerebral artery occlusion damage in the rat. Chin J Anesthesiol 2000; 20: 730-2.

11 Warner DS, Takaoka $S$, Wu B, et al.

Electroencephalographic burst suppression is not required to elicit maximal neuroprotection from pentobarbital in a rat model of focal cerebral ischemia. Anesthesiology 1996; 84: 1475-84.

12 Kawaguchi M, Kimbro JR, Drummond JC, Cole DJ, Kelly PJ, Patel PM. Isoflurane delays but does not pre 
vent cerebral infarction in rats subjected to focal ischemia. Anesthesiology 2000; 92: 1335-42.

13 Shuaib A, Murabit MA, Kanthan R, Howlett W, Wishart $T$. The neuroprotective effects of gamma-vinyl

GABA in transient global ischemia: a morphological

study with early and delayed evaluations. Neurosci Lett

1996; 204: 1-4.

14 Wilson JX, Gelb $A W$. Free radicals, antioxidants and neurologic injury: possible relationship to cerebral protection by anesthetics. J neurosurg anesthesiol (in press).

15 Cole DJ, Cross LM, Drummond JC, Patel PM, Jacobsen WK. Thiopentone and methohexital, but not pentobarbitone, reduce early focal cerebral ischemic injury in rats. Can J Anesth 2001; 48: 807-14. 